\title{
Comunicación
}

\section{Efecto de la utilización de subproducto de cervecería y sales minerales en vacas cruzadas en ordeño en el trópico peruano}

\author{
EFFECT OF THE USE OF BREWERY BY-PRODUCTS AND MINERAL SALTS IN DAIRY CROSSBRED \\ cows in the tropics of Peru
}

\author{
Roberto Del Águila L. ${ }^{1,3}$, César A. Reyes A. ${ }^{1}$, Wilder E. Suárez R. ${ }^{1}$, \\ Juan Rondón E. ${ }^{1}$, Alfredo Delgado C. ${ }^{2}$, Zoila Mirella Clavo P. ${ }^{1}$
}

\section{RESUMEN}

\begin{abstract}
El propósito del estudio fue evaluar la suplementación de residuo seco de cervecería (orujo) y sales minerales (Fosvimin ${ }^{\circledR}$ ) en la producción de leche de vacas cruzadas en la zona de Pucallpa, Perú. Se utilizaron tres grupos de seis vacas en diferente fase de lactación y de diverso grado de cruzamiento, pero con predominancia cebú. Los tratamientos fueron: $\mathrm{T} 1=$ pastura naturalizada $(\mathrm{PN}), \mathrm{T} 2=\mathrm{PN}+5 \mathrm{~kg}$ de orujo seco de cervecería (Os) y T3 $=\mathrm{PN}+\mathrm{Os}+100 \mathrm{~g} /$ vaca de sal mineral. Los tres grupos de vacas fueron enfrentados a los tres tratamientos por periodos de 10 días, en un diseño de bloques completos al azar, donde la producción de leche fue medida entre los días 6 y 10 de cada periodo. Los suplementos de T2 y T3 fueron proporcionados durante el ordeño. El forraje disponible de la pastura naturalizada fue de $2207.6 \mathrm{~kg} /$ ha de materia seca. La producción de leche en T1, T2 y T3 fue de 4.5, 4.3 y 4.8 1/vaca/día, respectivamente, habiendo diferencia significativa entre $\mathrm{T} 3$ con $\mathrm{T} 2$ y T1 $(\mathrm{p}<0.05)$. Los resultados mostraron un efecto benéfico de la suplementación combinada de residuos de cervecería y sales minerales en la producción de leche de vacas al pastoreo.
\end{abstract}

Palabras clave: cebuizado; sales minerales; orujo; pastura naturalizada

\section{Abstract}

The aim of the study was to evaluate the supplementation of dry brewery by-products and mineral salts (Fosvimin ${ }^{\circledR}$ ) in milk yield of crossbred cows in the Pucallpa area, Peru. Three groups of six cows each were used. Cows were at various phases of lactation and

\footnotetext{
${ }^{1}$ Estación Experimental del Trópico, Centro de Investigaciones IVITA, Universidad Nacional Mayor de San Marcos, Pucallpa, Perú

${ }^{2}$ Clínica de Animales Mayores, Facultad de Medicina Veterinaria, Universidad Nacional Mayor de San Marcos. Lima, Perú.

${ }^{3}$ E-mail: rdelaguilal@unmsm.edu.pe
}

Recibido: 21 de febrero de 2017

Aceptado para publicación: 30 diciembre de 2017 


\begin{abstract}
had various degree of crossbreeding, but with zebu predominance. The treatments were: $\mathrm{T} 1=$ naturalized pasture $(\mathrm{PN}), \mathrm{T} 2=\mathrm{PN}+5 \mathrm{~kg}$ of brewery residue $(\mathrm{Os})$ and $\mathrm{T} 3=\mathrm{PN}+\mathrm{Os}$ $+100 \mathrm{~g} / \mathrm{cow}$ of mineral salt. The three groups of cows were confronted with the three treatments for periods of 10 days, in a randomized complete block design, where daily milk yield was recorded between days 6 and 10 of each period. Supplements of T2 and T3 were provided during milking. The available forage of the naturalized pasture was 2207.6 $\mathrm{kg} / \mathrm{ha}$ of dry matter. The milk production in T1, T2 and T3 was 4.5, 4.3 and $4.81 /$ cow/day respectively, showing significant difference between $\mathrm{T} 3$ with $\mathrm{T} 2$ and $\mathrm{T} 1(\mathrm{p}<0.05)$. The results showed a beneficial effect of the combined supplementation of brewery's residues and mineral salts in the milk production of grazing crossbred cows.
\end{abstract}

Key words: zebu; mineral salts; brewery by-products; naturalized pasture

\section{INTRODUCCIÓN}

Los sistemas de producción en la Amazonía peruana se caracterizan por el empleo de ganado cruzado con diferente grado de mestizaje, pero con predominancia del genotipo cebú, manejados bajo un sistema semi-extensivo, con una alimentación basada principalmente en el uso de forraje al pastoreo (MINAGRI, 2008). Las pasturas están constituidas por pastos naturales (Axonopus compressus, Paspallum conjugatum y Homolepsis aturensis) y menos del 20\% corresponde a pastos mejorados o naturalizados como Brachiaria decumbens, Paspalum plicatulum (pasto negro) y algunas leguminosas (Araujo-Febres y Rodríguez, 2001; Ríos, 2007).

Una amplia gama de subproductos agroindustriales que tienen un adecuado potencial nutricional está disponible en grandes cantidades. El subproducto de cervecería (orujo) es un ejemplo típico. Se trata de un subproducto de la industria de la cerveza que utiliza granos de cereal como materia prima, donde el almidón es primariamente utilizado en el proceso de fabricación de la cerveza, quedando un residuo rico en proteínas (Senthilkumar et al., 2010). Tanto la forma fresca como seca son buenas fuentes de energía, proteína y fibra dietética (FAO, 2001; Mussatto et al., 2006); sin embargo, se debe tener cuidado con la cantidad suministrada para evitar problemas de desequilibrio ácidobase ruminal (Delgado et al., 2012).

Se registran valores de proteína cruda en un rango de $20 \%$ (Ranjhan, 1998) a $30 \%$ (Crickenberger y Johnson, 1982). La composición química y digestibilidad del orujo de cervecería seco en la zona de Ucayali fue descrita por Rosales y Tang (1996), reportando $16.4 \%$ de contenido proteico, $63.7 \%$ de carbohidratos solubles, 9.4\% de fibra cruda y una digestibilidad regular (37.95\%). Las variaciones en su composición se atribuyen al grano, a los métodos de obtención y a la naturaleza de los aditivos empleados. El residuo de cervecería puede ser explotado para emplearse como parte de la alimentación en animales de lechería en el trópico (Senthilkumar et al., 2010).

Un factor limitante para la producción de carne y leche es la baja calidad nutritiva de las gramíneas tropicales, que presentan bajos contenidos de proteína cruda (PC) (entre 3 y $10 \%$ ), digestibilidad inferior a $55 \%$ y carbohidratos no estructurales menores de 100 $\mathrm{g} / \mathrm{kg}$ de materia seca (MS) (Peruchena, 1999). Así mismo, el alto contenido de fibra limita el consumo voluntario al pastoreo, comprometiendo el nivel de respuesta animal (Del Águila, 2014). Otra seria limitación es la estacionalidad, ya que los niveles de producción forrajera son menores de $5 \mathrm{~kg} \mathrm{MS} / \mathrm{ha} /$ día en la época de menor precipitación (Araujo-Febres y Rodríguez, 2001). 
Un desequilibrio mineral en la dieta animal puede reducir la tasa de concepción y aumentar los abortos (Delgado et al., 2012), así como propiciar enfermedades metabólicas (Garmendia, 2006), incluyendo la ocurrencia de apetito depravado (pica) y fracturas (Salamanca, 2010). Los pastos y forrajes tropicales no satisfacen las necesidades de $\mathrm{Ca}$, $\mathrm{P}, \mathrm{Mg}, \mathrm{Zn}, \mathrm{Cu}, \mathrm{Mn}, \mathrm{Se}$, Co y I, como resultado de las limitaciones del suelo y clima, que impone restricciones nutricionales a los pastos (Salamanca, 2010). Por otro lado, el consumo de sales minerales mejora la condición corporal del ganado vacuno, aun cuando el consumo sea de pasturas de baja calidad nutritiva (Repetto et al., 2004).

Los forrajes de suelos tropicales son deficientes en macro y micro-minerales esenciales para el ganado, por lo que se requiere proveer suplementos minerales dietéticos con el objeto de promover una producción eficiente del ganado (McDowell et al., 1984). El Fosvimin ${ }^{\circledR}$ (Montana, Perú) es un suplemento mineral enriquecido con vitaminas $\mathrm{A}$, $\mathrm{D}_{3} \mathrm{y} \mathrm{E}, \mathrm{y}$ diversos minerales que son necesarios para cubrir los requerimientos nutricionales del ganado vacuno y de otras especies de producción (Suttle, 2010). Las vitaminas $\mathrm{A}, \mathrm{D}, \mathrm{E}, \mathrm{y}$ los minerales presentes en este compuesto intervienen en las defensas antioxidantes del organismo (NRC, 2001).

El presente estudio tuvo como objetivo evaluar el aporte del orujo y las sales minerales en la producción de leche de vacas cruzadas bajo pastoreo sobre pasturas naturalizadas, bajo las condiciones del trópico bajo peruano.

\section{Materiales y Métodos}

El trabajo se realizó en el Instituto Veterinario de Investigaciones Tropicales y de Altura (IVITA), sede Pucallpa, entre mayo y septiembre de 2013. La estación experimental se encuentra ubicada en el distrito de Campo Verde, provincia de Coronel Portillo, región Ucayali (Perú), a 266 msnm. La zona presenta una temperatura media de $26.7^{\circ} \mathrm{C}$, precipitación pluvial entre 79 y $642 \mathrm{~mm} / \mathrm{mes}$, humedad relativa media de $85 \%$ y suelo franco-arcillo-arenoso con $\mathrm{pH}$ 4.9. El ecosistema es Bosque Tropical Semi-siempre Verde Estacional (Díaz, 1999).

Antes de iniciar el estudio, se realizó una evaluación de la disponibilidad de forraje, utilizando el método del rendimiento comparativo de peso seco, propuesto por Haydock y Shaw (1975), encontrando Brachairia decumbens, Desmodium ovalifolium y malezas con 21, 30 y $49 \%$ de participación en la pastura respectivamente.

Se seleccionaron 18 vacas en ordeño, con cruces indeterminados de Bos taurus con Bos indicus, pero con predominancia cebú (Cuadro 1). Como criterio de exclusión se consideró vacas en el último tercio de lactación. Las vacas del estudio permanecieron juntas, pero se formaron tres grupos de seis vacas en forma aleatoria: T1: pastura naturalizada sola $(\mathrm{PN}) ; \mathrm{T} 2: \mathrm{PN}+5 \mathrm{~kg}$ de orujo seco de cervecería (Os); T3: PN + Os $+100 \mathrm{~g}$ de sal mineral/vaca. La composición de la sal mineral se muestra en el Cuadro 2.

El orujo tenía $9 \%$ de proteína cruda. No se proporcionó otro tipo de sal durante el estudio. Así mismo, se dejó de proporcionar el polvillo de arroz que usualmente recibían las vacas cinco días antes de iniciar las evaluaciones. El ordeño fue manual una vez al día, a partir de las 04:00 h. La producción de leche de cada vaca fue pesada en una balanza tipo reloj. Las producciones registradas entre el $6^{\circ}$ y $10^{\circ}$ día de ordeño fueron consideradas en el análisis del estudio.

Cada grupo de vacas participó en forma rotatoria por 10 días en cada uno de los tratamientos, por lo que una vuelta duraba 30 días. Se hizo tres vueltas en total; la primera en mayo y se dejó un mes de descanso, la segunda vuelta se hizo en julio y se descansó otro mes, y la tercera vuelta se hizo en setiembre. Las vacas se mantuvieron al pastoreo con una carga animal de $2.3 \pm 0.2 \mathrm{UA} / \mathrm{ha}$. 
Cuadro 1. Características del hato en el que se midió la aplicación de orujo seco y sal mineral ( $\mathrm{n}=6$ vacas por grupo)

\begin{tabular}{cccccc}
\hline Grupos & $\begin{array}{c}\text { Edad } \\
\text { (años) }\end{array}$ & N. ${ }^{\circ}$ de partos & $\begin{array}{c}\text { Lactación } \\
\text { (meses) }\end{array}$ & $\begin{array}{c}\text { Condición } \\
\text { corporal }\end{array}$ & $\begin{array}{c}\text { Peso } \\
(\mathrm{kg})\end{array}$ \\
\hline I & $8.7 \pm 2.0$ & $4.2 \pm 1.3$ & $2.7 \pm 1.4$ & $3.1 \pm 0.4$ & $401.0 \pm 28.2$ \\
II & $8.6 \pm 1.4$ & $4.0 \pm 1.3$ & $3.3 \pm 1.4$ & $3.2 \pm 0.3$ & $492.3 \pm 45.4$ \\
III & $7.5 \pm 2.6$ & $3.3 \pm 1.5$ & $2.5 \pm 1.4$ & $3.1 \pm 0.5$ & $426.8 \pm 48.8$ \\
\hline
\end{tabular}

Cuadro 2. Composición de la sal mineral (por kg)

\begin{tabular}{lr}
\hline Vitamina A & $320,000 \mathrm{UI}$ \\
Vitamina D3 & $48,000 \mathrm{UI}$ \\
Vitamina E & $320 \mathrm{UI}$ \\
Calcio & $200 \mathrm{~g}$ \\
Fósforo & $100 \mathrm{~g}$ \\
Magnesio & $21 \mathrm{~g}$ \\
Cobre & $320 \mathrm{mg}$ \\
Cobalto & $5 \mathrm{mg}$ \\
Hierro & $1,600 \mathrm{mg}$ \\
Manganeso & $280 \mathrm{mg}$ \\
Selenio & $5 \mathrm{mg}$ \\
Yodo & $32 \mathrm{mg}$ \\
Zinc & $1,280 \mathrm{mg}$ \\
Excipientes c.s.p. & $1 \mathrm{~kg}$ \\
\hline
\end{tabular}

Al inicio del ordeño, cada vaca de T2 recibió $5 \mathrm{~kg}$ de orujo seco y cada vaca de $\mathrm{T} 3$ recibió una mezcla $5 \mathrm{~kg}$ de orujo $+100 \mathrm{~g}$ de sal mineral. La disponibilidad de agua fue permanente.

El diseño experimental empleado fue el de Bloques Completos al Azar, donde los bloques fueron los grupos de vacas y cada vaca fue una repetición. Se utilizó la prueba de Duncan $(p<0.05)$ para determinar diferencias entre tratamientos.

\section{Resultados}

Se tuvo una mayor disponibilidad de forraje al inicio del pastoreo, habiendo una disminución de $29.8 \%$ de forraje al final del mismo (Cuadro 3)

El suplemento del orujo seco de cervecería (T2) no fue suficiente para mejorar la producción de leche comparada con la producción de T1 en solo pastura; sin embargo, la adición combinada de orujo y sal mineral (T3) logró un incremento significativo de 11.1 y $6.7 \%$ comparado con orujo seco y pastura naturalizada, respectivamente ( $\mathrm{p}<0.05$, Cuadro 4).

\section{Discusión}

Producir leche en condiciones de trópico bajo sigue siendo un reto en el desarrollo de la ganadería lechera peruana, pues pese a poderse superar las condiciones ambientales con tecnología, esta no llega a los ganaderos de pequeña escala, quienes siguen haciendo uso de las pasturas naturales con escaso nivel nutricional (Araujo-Febres y Rodríguez, 2001; Ríos, 2007). Además, las pasturas se van degradando año a año, sin que se tome en cuenta una alternativa válida para detener 
Cuadro 3. Disponibilidad de forraje (MS kg/ha) en una pastura naturalizada en Pucallpa, Perú

\begin{tabular}{lcc}
\hline \multirow{2}{*}{ Componentes } & \multicolumn{2}{c}{ Disponibilidad $(\mathrm{kg} / \mathrm{ha} \mathrm{MS})$} \\
\cline { 2 - 3 } & Inicio & Final \\
\hline Brachiaria decumbens & 599.5 & 338.8 \\
Leguminosas & 908.1 & 501.8 \\
Malezas & $1,086.8$ & 980.2 \\
\hline Materia seca (MS) total & $2,594.4^{\mathrm{a}}$ & $1,820.8^{\mathrm{b}}$ \\
\hline
\end{tabular}

${ }^{a, b}$ Letras diferentes dentro de filas indican diferencia significativa $(p<0.05)$

Cuadro 4. Producción de leche (1/vaca/día) de vacas cruzadas con predominancia cebú en pasturas naturalizadas, suplementadas con residuos de cervecería y sal mineral, en las condiciones del trópico bajo peruano $(\mathrm{n}=6$ por grupo)

\begin{tabular}{lcccc}
\hline \multirow{2}{*}{ Tratamientos } & \multicolumn{3}{c}{ Grupos de vacas } & \multirow{2}{*}{ Promedio } \\
\cline { 2 - 4 } & 1 & 2 & 3 & \\
\hline Pastura naturalizada (PN) & 4.5 & 4.2 & 4.2 & $4.3 \pm 0.2^{\mathrm{b}}$ \\
PN + orujo seco de cervecería & 3.9 & 4.3 & 4.4 & $4.2 \pm 0.3^{\mathrm{b}}$ \\
PN + orujo seco + sales minerales & 4.9 & 4.9 & 4.7 & $4.8 \pm 0.1^{\mathrm{a}}$ \\
\hline
\end{tabular}

${ }^{a, b}$ Superíndices diferentes dentro de columna indican diferencia significativa $(p<0.05)$

este proceso. Queda claro que las pasturas en la Amazonía están constituidas por especies nativas (Axonopus compressus, Paspallum conjugatum y Homolepsis aturensis) y menos del $20 \%$ corresponde a pastos mejorados o naturalizados como Brachiaria decumbens y Paspalum plicatulum, este último denominado pasto negro (que no ha tenido el éxito esperado en términos nutricionales y de palatabilidad), así como algunas leguminosas (Araujo-Febres y Rodríguez, 2001; Ríos, 2007).

La mejora esperada en la producción de leche con el solo aporte del orujo de cervecería no se pudo observar en el presente trabajo, pues posiblemente se requeriría haber tenido un mayor periodo de acostumbramiento y haber suministrado cantidades mayores; sin embargo, se observó un incremento significativo de la producción de leche con el consumo de orujo + sal mineral, donde este último aportó vitaminas y micronutrientes (Salamanca, 2010). El selenio orgánico de la sal mineral posiblemente haya mejorado el funcionamiento enzimático y el transporte de sustancias a través del organismo de los animales, permitiéndoles tener una mejor respuesta productiva (Salamanca, 2010; Repetto et al., 2004).

La ausencia de una mejora productiva con el suplemento del orujo pudo deberse al reducido aporte de PC, el cual no llega a mejorar el aporte de la gramínea naturalizada. Roca (1998) sostiene que la menor producción de leche obtenida con gramíneas tropicales ha sido atribuida a limitaciones en el consumo de nutrientes digestibles, particularmente energía. En vacas lactantes que consumen gramíneas tropicales la energía es aún más limitante que la $\mathrm{PC}$ para la producción de leche (Pezo et al., 1992). 
Los subproductos de la agroindustria pueden cumplir un rol importante en la alimentación animal pues, así como se tiene el orujo de cervecería, se tiene el derivado del procesamiento de la palma aceitera (palmiste), caña de azúcar, arroz y otros. No obstante, se requiere, además, de un cambio de actitud del ganadero para la mejora de las pasturas existentes. Por otro lado, la adición de micronutrientes a la dieta diaria de las vacas mostró un efecto positivo en la producción de leche, lo cual confirma la deficiencia de minerales en los animales de la zona.

Los niveles de producción de leche coinciden con Reátegui et al. (1995), quienes reportan $3.0 \mathrm{l} / \mathrm{vaca} / \mathrm{dí}$ e en fincas de pequeños productores con vacas consumiendo $B$. decumbens en monocultivo en la zona de Pucallpa. Asimismo, Vera et al. (1996) reportaron bajo las condiciones de trópico seco para la época lluviosa y en pasturas de $B$. decumbens en la La Libertad, Colombia, producciones de 4.0 1/vaca/día. En cambio, De la Torre (citado por Pezo et al., 1992), en el rebaño del Centro de Investigación IVITAPucallpa, reportó una producción diaria de $8.61 /$ vaca, lo que se explica por tratarse de épocas diferentes de explotación pecuaria, en la que se tuvo en cuenta mejores niveles de manejo y alimentación de las vacas lecheras.

\section{Conclusiones}

El suplemento de $5 \mathrm{~kg}$ de orujo seco de cervecería no incrementó la producción de leche en vacas al pastoreo sobre pasturas naturalizadas en la zona del trópico bajo del Perú; sin embargo, el suplemento de $100 \mathrm{~g}$ de sal mineral $+5 \mathrm{~kg}$ de orujo seco de cervecería aumentó significativamente $(\mathrm{p}<0.05)$ la producción de leche.

\section{Literatura Citada}

1. Araujo-Febres O, Rodríguez N. 2001. La amonificación de henos como téc- nica para mejorar su aprovechamiento. Rev Inv Vet Perú Supl 1: 88-91.

2. Crickenberger RG, Johnson BH. 1982. Effect of feeding wet brewer's grains to beef heifers on wintering performance, serum selenium and reproductive performance. J Anim Sci 54: 18-22. doi:10.2527/jas1982.54118x

3. Del Aguila R. 2014. Evaluación de tres tipos de pasturas y su efecto en el nivel de nitrógeno ureico en leche de vacas bajo pastoreo en Pucallpa. Tesis de Magíster. Lima: Univ. Nacional Agraria La Molina. 83 p.

4. Delgado A, Trigueros A, Tang T, Angelats $R$, Gavidia C. 2012. Efecto de un modificador orgánico en la ganancia de peso en ganado cebú en el trópico peruano. Rev Inv Vet Perú 23: 153-159. doi: 10.15381/rivep.v23i2.895

5. Díaz E. 1999. Ecosistema amazónico con énfasis en la amazonia peruana. Características, potencial y riesgos de uso. Pucallpa, Perú: Univ. Nacional de Ucayali. $54 \mathrm{p}$.

6. [FAO] Organización de las Naciones Unidas para la Agricultura y la Alimentación. 2001. Uso del ensilaje en el trópico, privilegiando opciones para pequeños campesinos. Memorias Conferencia Electrónica de la FAO sobre Ensilajes en el Trópico. [Internet]. Disponible en: http://www.fao.org/3/ax8486s.pdf

7. Haydock KP, Shaw NH. 1975. The comparative yield method for estimating dry matter yield of pastures. Aust J Exp Agric Anim Husb 15: 663-670.

8. [NRC] National Research Council. 2001. Nutrient requirements of dairy cattle. $7^{\text {th }}$ revised ed. USA: National Academy Press. 408 p.

9. McDowell LR, Conrad JH, Ellis GL, Loosli JK. 1984. Minerales para rumiantes en pastoreo en regiones tropicales. Florida, USA: Universidad de Florida. $92 \mathrm{p}$.

10. [MINAGRI] Ministerio de Agricultura. 2008. Especial IV CENAGRO. [Internet]. Disponible en: http://www.minagri.gob.pe/ 
11. Montana. 2017. Sales minerales, disponible em: http://montana.perulactea.com/productos-veterinarios/salesminerales/fosvimin-p-100/

12. Mussatto SI, Dragone G, Roberto IC. 2006. Brewers' spent grain: generation, characteristics and potential applications. J Cereal Sci 43: 1-14. doi: 10.1016/ j.jcs.2005.06.001

13. Peruchena CO. 1999. Suplementación de bovinos para carne sobre pasturas tropicales. Aspectos nutricionales, productivos y económicos. En: XXXVI Congreso Anual de la Sociedad Brasilera de Zootecnia. Porto Alegre, Brasil.

14. Pezo D, Romero F, Muhammad I. 1992. Producción, manejo y utilización de los pastos tropicales para la producción de leche y carne. En: FernándezBaca S (ed). Avances en la producción de leche y carne en el trópico americano. Turrialba, Costa Rica: Centro Agronómico Tropical de Investigación y Enseñanza (CATIE). p 47-98.

15. Ranjhan SK. 1998. Nutritient requirements of livestock and poultry. New Delhi, India: Indian Council of Agricultural Research,. $72 \mathrm{p}$.

16. Reátegui $K$, Vera $R$, Loker WL, Vásquez M. 1995. On farm grasslegume pasture performance in the Peruvian rainforest. Exp Agric 31: 227240. doi: 10.1017/S0014479700025308

17. Repetto J, Donovan A, García F. 2004. Carencias minerales, limitantes de la producción. Sitio Argentino Producción Animal [Internet]. Disponible en: http:// www.produccion-animal.com.ar/ suplementacion_mineral/ 18_carencias_limitantes_produccion.pdf
18. Ríos J. 2007. Enfoques integrales de producción ganadera en la Amazonía peruana. En: XX Reunión Asociación Latinoamericana de Producción Animal. Cusco, Perú.

19. Roca PF. 1998. Niveles de fertilización en el establecimiento y producción de una asociación Arachis pintoi CIAT 18744 y Brachiaria decumbens CIAT 606 en Pucallpa. Tesis de Ingeniero Agrónomo. Pucallpa, Perú: Univ. Nacional de Ucayali. $42 \mathrm{p}$.

20. Rosales JM, Tang T. 1996. Composición química y digestibilidad de insumos alimenticios de la zona de Ucayali. IIAPFolia Amazónica 8(2): 13-27.

21. Salamanca A. 2010. Suplementación de minerales en la producción bovina. REDVET 11(9) [Internet]. Disponible en: http://www.veterinaria.org/revistas/ redvet/n090910/091009.pdf

22. Senthilkumar S, Viswanathan TV, Mercy AD, Gangadevi P, Ally K, Philomina PT. Tamilnadu J. 2012. Economics of feeding brewery waste in lactating cows. Tamilnadu J Vet Anim Sci 8: 286-289.

23. Senthilkumar S, Viswanathan TV, Mercy AD, Gangadevi P, Ally K, Shyama K. 2010. Chemical composition of brewery waste. Tamilnadu J Vet Anim Sci 6: 49-51.

24. Suttle N. 2010. Minerals nutrition of livestock. $4^{\mathrm{a}}$ Ed. CABI-Cambridge. $608 \mathrm{p}$.

25. Vera RR, García O, Botero R, Ullrich $C$. 1996. Producción de leche y reproducción en sistemas de doble propósito: algunas implicaciones para el enfoque experimental. Pasturas Tropicales 18(3): 25-32. 